\title{
Rating the State Government Public Pension Plans in the US
}

\author{
Jiapeng Liu ${ }^{1}$, Rui $\mathrm{Lu}^{2}$ \& Zhengyang Zhang ${ }^{3}$ \\ ${ }^{1}$ School of Economics and Management, China Jiliang University, Hangzhou, China \\ ${ }^{2}$ Lingnan (University) College, Sun Yat-Sen University, Guangzhou, China \\ ${ }^{3}$ Industrial and Commercial Bank of China, Jinan, China \\ Correspondence: Rui Lu, Lingnan (University) College, Sun Yat-sen University, No. 135, Xingang Xi Road, \\ Guangzhou, China, 510275. Tel: 86-20-84112053.E-mail:lurui@mail.sysu.edu.cn
}

Received: September 10, 2015

doi:10.5430/afr.v4n4p39
Accepted: September 25, 2015

Online Published: October 1, 2015

\begin{abstract}
The government-sponsored pension plans in the US, mostly defined benefit (DB) pension plans, are severely underfunded. In this study we examine the current state of the public pension plans, and rate them based on their actuarial funding ratio, risky asset allocation, and other multiple variables using both a simple ranking and a principal component analysis method. We aim to help public employees/retirees understand the financial health of their pension plans, and to raise the public awareness of the pension Tsunami.
\end{abstract}

Keywords: Public pension, Funding ratio, Asset allocation risk

JEL Codes: G18, G11, G28

\section{Introduction}

The 50 states across the U.S. are currently facing a big issue with their government-sponsored pension plans. These plans, mostly defined benefit (DB) pension plans, are severely underfunded; that is, their assets are not sufficient to meet future liabilities (i.e., employees' retirement payment and benefit). The total funding gap, or the difference between pension assets and liabilities, is estimated to be $\$ 843$ billion as of March 2013 for 99 pension systems. (Note 1) Using a more conservative method to estimate pension liabilities, some economists (i.e., Novy-Marx and Rauh, 2010, 2011; Rauh, 2011) estimate the funding gap to be $\$ 4.4$ trillion. (Note 2) As a reference point, these unfunded pension liabilities of $\$ 4.4$ trillion accounts for $33 \%$ of the 2011 U.S. real gross domestic product (GDP) of \$13.3 trillion (Healey, Hess, and Nicholson, 2012).

Table 1. Five states in the U.S. with the largest pension funding shortfall

Panel A: If using actuarial rate to discount future pension liabilities

\begin{tabular}{lll}
\hline State & Pension funding shortfall (\$ bil.) & Shortfall as a \% of total pension liabilities \\
\hline California & 154.2 & $32 \%$ \\
Illinois & 85.4 & $57 \%$ \\
Ohio & 75.3 & $39 \%$ \\
New Jersey & 62.9 & $51 \%$ \\
Texas & 53.7 & $30 \%$ \\
\hline
\end{tabular}

Panel B: If using a conservative estimation method, as suggested by Novy-Marx and Rauh (2009)

\begin{tabular}{lll}
\hline State & Pension funding shortfall (\$ bil.) & Shortfall as a \% of total pension liabilities \\
\hline California & 475.7 & $59 \%$ \\
Illinois & 219.1 & $77 \%$ \\
Ohio & 216.9 & $65 \%$ \\
Texas & 188.2 & $60 \%$ \\
New York & 166.4 & $47 \%$ \\
\hline
\end{tabular}

Table 1 lists top (worst) five states with the largest pension funding shortfall as of fiscal year of 2009, based on a study conducted by Novy-Marx and Rauh (2009). When using actuarial rate to discount future pension liabilities, California suffers the largest pension funding gap of $\$ 154.2$ billion, followed by Illinois ( $\$ 85.4$ billion), Ohio ( $\$ 75.3$ billion), New 
Jersey ( $\$ 62.9$ billion), and Texas ( $\$ 53.7$ billion). However, when using Treasury yield as a beach market to discount future pension liabilities, the funding gap for California widens to $\$ 475.7$ billion. When considering funding gap as a percentage of total pension liabilities, Illinois is the worst state with an underfunding ratio of $57 \%$ (when using actuarial rate as a discount rate) or $77 \%$ (when using Treasury yield as a bench mark).

The severe underfunding of public pension plans has threatened the retirement security of a large population, as public pension plans cover pension benefits for about 12.8 million active public employees and 5.9 million retirees and other annuitants. (Note 3) Rauh (2010) estimates that at an aggregate level, the pension payment would exhaust pension assets by 2028 , and that several pension plans would run out much sooner - Illinois would run out of pension assets in 2018, and New Jersey, Connecticut and Indiana follow suit during the next year.

More severely, the 50 state governments continue to face major fiscal challenges and budget deficits. The combined budget gaps were $\$ 350$ billion for 2010 and 2011, making most state governments unable to make pension contributions and to narrow funding gap. Facing various fiscal constraints and budget difficulties, some states have to cut spending, withdraw from reserves, or reduce investments in public services. In fact, according to the Center on Budget and Policy Priorities (2012), the budget difficulties have led at least 30 states to raise taxes, in some cases quite substantially. For example, lawmakers of Illinois, one of the states with the largest pension funding gap (as shown in Table 1), passed a big income-tax increase in January 2011, with the individual income-tax rate jumping to 5\% from $3 \%$ and the corporate tax jumping to $7 \%$ from $4.8 \%$. Illinois lawmakers hope that the tax increase will help to close the pension funding shortfall and reduce the budget deficit (Bellandi, 2011). (Note 4) According to Novy-Marx and Rauh's (2009) calculation, if the governments elect to raise the tax to close the funding gap between $\$ 1.27$ to $\$ 3.26$ trillion, each household would need to contribute an additional tax payment of $\$ 21,500$. The effect of underfunded pension plans therefore touches a large percentage (if not all) of the population in the country, including those at risk and the taxpayers who may be ultimately called upon to close the funding gap (Mohan and Zhang, 2014).

The primary objective of this paper is to provide the first comprehensive study on the current state of public pension plans in the US. In particular, we rate each state pension plan based on a thorough evaluation of plan financial health (from A, A-,.., to D, F). Our rating framework will consider pension funding levels, investment risks, state fiscal constraints, and workforce/retirees demographics, as well as other important factors. We aim to help public employees/retirees understand the financial health of their pension plans, and to raise the public awareness of the pension Tsunami.

The remainder of this paper is organized as follows. Section 2 discusses the magnitude of pension funding gap. Section 3 describes the data, variables and methodology. Section 4 shows the empirical results. Section 5 concludes.

\section{The Magnitude of Pension Funding Gap}

Compared to the studies on the private pension plans, the studies concerning state government public pension plans have been sparse for a fairly long time. The lack of the related studies could be due to the different orientation of issues as well the data availability (Mohan and Zhang, 2012). In particular, firms or sponsors of private pension plans, including those firms listed on the stock exchanges, are required to file financial statements and other reports (i.e., Form 5500) to their shareholders and regulatory and government agencies (i.e., the Securities Exchange Commissions, Department of Labor, Pension Benefit Guaranty Corporation, and Internal Revenue Service). But the pertinent law and regulations on public pension plans are generally nonexistent. (Note 5) Although state governments are required to make their Comprehensive Annual Financial Report (CAFR) and budget report publicly available online, the information and discussions on the public pension plans in these reports is very limited. Indeed, the lack of information transparency for state and local governments has become a severe concern for the general public, municipal bond investors, and lawmakers. It has been reported that one third of the governments that issued debt to the public failed to disclose their financial information from 2005 to 2007. (Note 6)

This paper is mainly related with previous studies on public pension underfunding magnitude/scope. Novy-Marx and Rauh have been active researchers in public pension plans and they have conducted a series of the studies on the size and scope of public pension obligations. Using data of the 116 largest public pension plans in 2008, Novy-Marx and Rauh (2009) estimate the total pension liabilities to be $\$ 2.97$ trillion for the 50 states in 2008 . For the first time, this article has cautioned the general public of the staggering magnitude of public pension shortfall. In another article, Novy-Marx and Rauh (2010) conservatively estimate "already-promised" benefits between $\$ 3.21$ and $\$ 5.2$ trillion, depending on the discount assumption to be used. The latest estimation of total pension liabilities by Rauh (2011) is as high as $\$ 7.03$ trillion. The growth of pension obligations is largely due to continuous decrease of Treasury yields, which is used to discount future pension payments. Regarding the effect of unfunded pension liabilities on the social welfare, Novy-Marx and Rauh (2009) report that in order to close funding gap, which is between $\$ 1.27$ and \$3.26 
trillion, each household would need to make an additional tax payment of $\$ 21,500$. Furthermore, assuming that newly created pension benefit debt is funded, Rauh (2010) estimates that the existing pension payment would exhaust pension assets, in aggregate, by 2028 .

Several large consulting firms and research institutions have recently focused on public pension issues as well. Using data in fiscal year 2009, the Pew Center on the States (2011) estimates the total pension liabilities to be $\$ 2.94$ trillion. The Pew Center uses the actuarial assumption, rather than Treasury yields as a discount rate to estimate pension liabilities, so its estimation of pension liabilities is smaller than that made by Novy-Marx and Rauh. The total pension liabilities would jump to $\$ 4.6$ trillion if the Treasury rate is used to discount the same liabilities by the Pew Center. The total size of pension liabilities for the 126 largest public plans in 2010 estimated by Wilshire (2012) is $\$ 3.23$ trillion. According to Public Fund Survey (2013), the aggregate pension liabilities are $\$ 3.49$ trillion and pension assets exceed $\$ 2.65$ trillion, with a funding deficit of $\$ 843$ billion. The severe underfunding of public pension plans has threatened the retirement security of a large population. Moreover, taxpayers may be ultimately called upon to close the funding gap, as the state governments continue to face major fiscal challenges and budget deficits.

\section{Data, Variables, and Methodology}

The data on public pension funds are obtained from the Public Plans Database (PPD), maintained by the Center for Retirement Research at Boston College. The sample period extends from fiscal years 2001 through 2011, covering 126 pension systems for 50 states and the District of Columbia. These pension systems together held $\$ 2.28$ trillion pension assets at the end of fiscal year 2011. In addition, the public employee union membership and coverage data are obtained from the Union Membership and Coverage Database. (Note 7)

The key variables in this study are pension funding ratio and risky asset allocation. Pension funding ratio (FUNDING_RATIO) is defined as the ratio of pension actuarial assets over pension actuarial liabilities.

$$
\text { FUNDING_RATIO }=\frac{\text { PENSION ACTUARIAL ASSETS }}{\text { PENSION ACTUARIAL LIABILITIES }}
$$

Risky asset allocation is the percentage of a pension plan assets invested in equity market and alternatives (i.e., private equity and venture capital). Other variables including (1) \% of annual required contribution paid, (2) 1-year actual return of pension assets, (3) actuarial discount rate, (4) projected total annual required Contribution as a $\%$ of payroll, (5) total normal cost as a \% of payroll, (6) inflation rate assumption, (7) active to retired employee ratio, and (8) \% of unionized employees.

Table 2. Anticipated rating structure of public pension plans

\begin{tabular}{lll}
\hline Rating Classification & Cardinal Value & Letter Grade \\
\hline Safe pension plans & & \\
\hline Highest Grade & 1 & $\mathrm{~A}$ \\
High Grade & 2,3 & $\mathrm{~A}-, \mathrm{B}+$ \\
Medium Grade & 4,5 & $\mathrm{~B}, \mathrm{~B}-$ \\
\hline At-risk pension plans & & \\
\hline Low Grade & $6,7,8$ & $\mathrm{C}+, \mathrm{C}, \mathrm{C}-$ \\
Poor Standing & 9,10 & $\mathrm{D}, \mathrm{F}$ \\
\hline
\end{tabular}

This table is created based on Jorion, Liu and Shi (2005). We rate all pension plans from fiscal year 2001 to 2011 using (1) actuarial funding ratio, (2) risky asset allocation, and (3) a combination of 10 variables as ranking variables. When ranking pension plans based on multivariate variables, we use a simple ranking and a principal component analysis (PCA) method. The pension plan rating structure is similar to bond credit ratings performed by S\&P. The rating structure is shown inTable2. We classify all pension plans into two categories: safe pension plans and at-risk pension plans. Safe pension plans refer to those with a rating between B- to A and at-risk plans refer to those with a rating between $\mathrm{F}$ to $\mathrm{C}+$.

\section{Empirical Results}

In this section we first describe summary statistics for all pension plans during the fiscal years of 2001 to 2011 . We then proceed to rate all pension funds based on various criteria. 


\subsection{Summary Statistics}

Table 3. Summary Statistics

\begin{tabular}{|c|c|c|c|c|c|c|c|}
\hline Variable Name & Mean & MIN & Q1 & Median & Q3 & MAX & STD \\
\hline Actuarial Funding Ratio & 83.3386 & 19.0786 & 72.6153 & 84.2485 & 96.3889 & 147.7331 & 17.2269 \\
\hline Risky Asset Allocation \% & 58.9846 & 0.0000 & 54.0000 & 60.5000 & 66.0000 & 88.0000 & 11.0079 \\
\hline $\begin{array}{l}\% \text { of Annual Required } \\
\text { Contribution Paid }\end{array}$ & 92.3146 & 0.0000 & 79.5900 & 100.0000 & 100.0000 & 1727.7000 & 59.0401 \\
\hline $\begin{array}{l}\text { 1-Year Actual Return of } \\
\text { Pension Assets }\end{array}$ & 5.6359 & -29.6300 & -4.4750 & 9.3000 & 15.0100 & 36.2400 & 12.3640 \\
\hline $\begin{array}{l}\text { Actuarial Rate (Pension } \\
\text { Liab. Discount Rate) }\end{array}$ & 0.0798 & 0.0450 & 0.0775 & 0.0800 & 0.0825 & 0.0900 & 0.0039 \\
\hline $\begin{array}{l}\text { Projected Total Annual } \\
\text { Required Contribution as } \\
\text { a \% of Payroll }\end{array}$ & 19.3656 & 0.0000 & 13.5900 & 17.7500 & 24.3600 & 100.1100 & 9.0284 \\
\hline $\begin{array}{l}\text { Total Normal Cost as a \% } \\
\text { of Payroll }\end{array}$ & 12.4826 & -19.2700 & 9.8500 & 11.5435 & 14.6400 & 49.7600 & 4.7384 \\
\hline Inflation Rate Assumption & 0.0358 & 0.0050 & 0.0300 & 0.0350 & 0.0400 & 0.3000 & 0.0103 \\
\hline $\begin{array}{ll}\text { Active to } & \text { Retired } \\
\text { Employee Ratio } & \\
\end{array}$ & 3.3255 & 0.0376 & 1.8689 & 2.2712 & 2.8239 & 179.7286 & 7.7921 \\
\hline $\begin{array}{ll}\% \text { of } & \text { Employees } \\
\text { w/Collective } & \text { Bargaining } \\
\text { Contract } & \end{array}$ & 0.3962 & 0.1038 & 0.2297 & 0.3679 & 0.5587 & 0.7533 & 0.1766 \\
\hline
\end{tabular}

Table 3 shows that public pension plans, on average, are underfunded during our sample period. A historical evolution of pension funding status is illustrated in Figure 1. The mean (median) actuarial funding ratio is $83.34 \%$ (84.25\%), and half of the pension plans have a funding ratio is between $72.62 \%$ and $96.39 \%$. The minimum and maximum funding ratios are $19.08 \%$ and $147.73 \%$, with a standard deviation of $17.23 \%$, indicating a high variability of pension funding status. Pension funds tend to invest a majority of their assets in risky assets, including equity and alternatives. Table 3 shows that $75 \%$ of the plans in the sample have allocated more than $54 \%$ of their pension funds into risky assets. The average (median) risky asset allocation is $58.98 \%(60.5 \%$ ), with a range of 0 to $88 \%$ and a standard deviation of $11.01 \%$. Note that about half of the pension plans did not report their actuarial assets and liabilities in 2011 when we obtain the data. In Figure 2, we provide average and median risky asset allocation for the pension funds from 2001 to 2011. The average risky asset allocation peaked in $2004-2006$ at about $62 \%$ and then declined to $56 \%$ in 2009 . Risky asset allocation is about 57\% at the end of fiscal year 2011. Note that about half of the pension plans did not report their asset allocation in 2011 when we obtain the data.

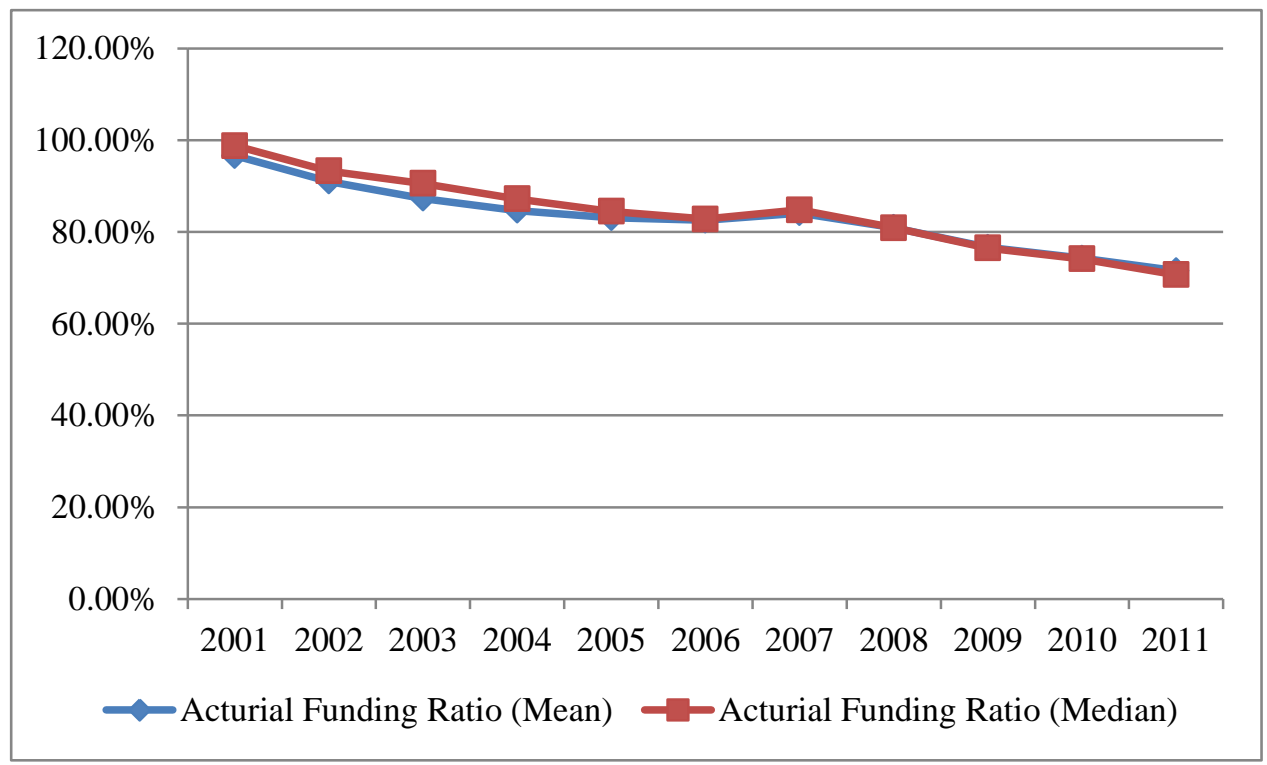

Figure 1. Average and Median Pension Actuarial Funding Ratios for US Public Funds from 2001 to 2011 


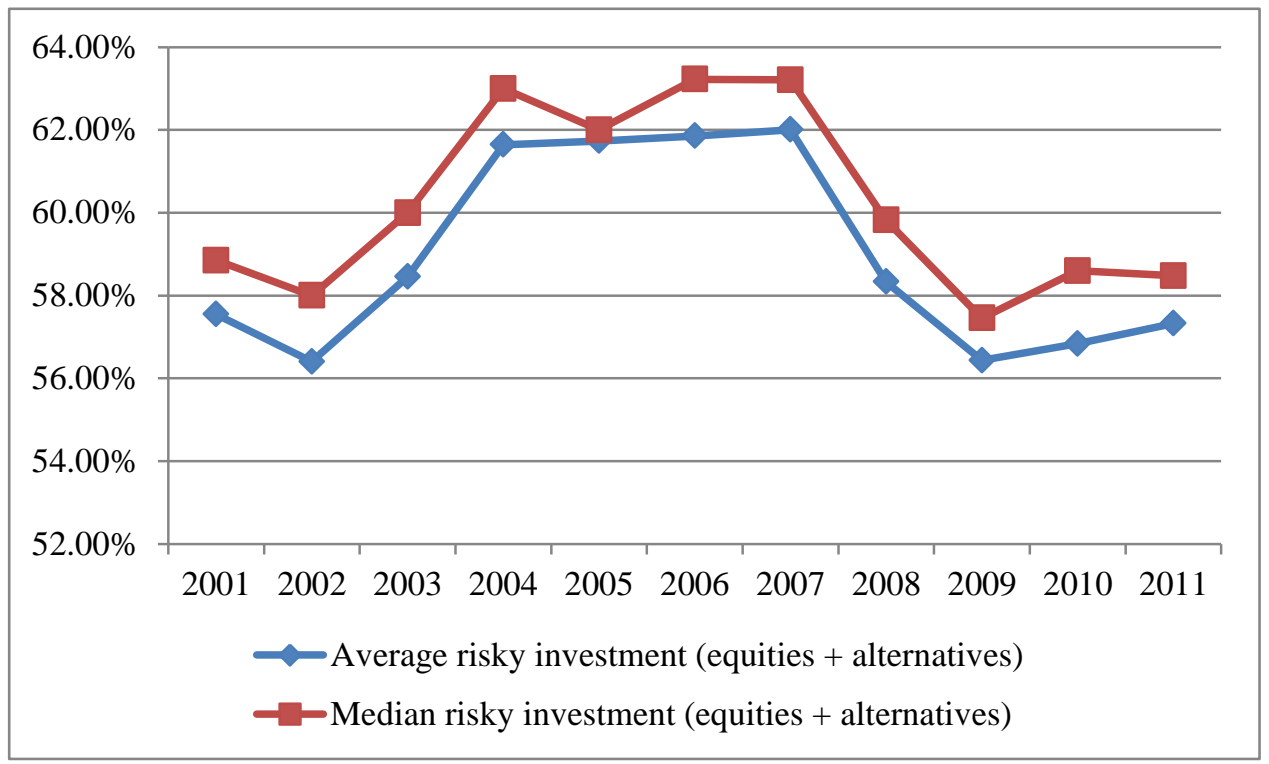

Figure 2. Average and Median Risky Asset Allocation for US Public Funds from 2001 to 2011

These two figures are created based on the authors' own calculations. And they are consistent with the figures reported by Mohan and Zhang $(2012,2014)$. As shown in Table 3, the mean actuarial rate is $7.98 \%$, with a standard deviation of $0.39 \%$. Actuarial rate is based on pension fund long-term expected investment returns. Such a relatively low variation probably indicates that plans do not often change their pension discount rate. In contrast to the actuarial rate, the actual investment returns are lower. The average 1-year investment return is $5.64 \%$, employing that pension plans generally underperform their expectations by $2.34 \%$. Table 3 shows there are relatively few working employees (3.33) for each retiree, an indication of an aging workforce. Finally, on average $39.62 \%$ of all public employees are unionized.

\subsection{Rating Pension Plans}

We use (1) actuarial funding ratio, (2) risky asset allocation percentage, and (3) a combination of 10 variables as ranking variables to rate all pension plans.

\subsubsection{Ranking Pension Plans by Actuarial Funding Ratios}

Table 4. Ranking Pension Plans by Actuarial Ratio

\begin{tabular}{|l|l|l|l|l|}
\hline$(1)$ & $(2)$ & $(3)$ & $(4)$ & $(5)$ \\
\hline Fiscal Year & Pension Name & Actuarial Funding Ratio (\%) & Ranking & Letter Rating \\
\hline 2010 & Washington LEOFF P & 126.5768 & 1 & A \\
\hline 2010 & New York State Tea & 100 & 2 & A \\
\hline 2010 & NY State \& Local E & 100 & 2 & A \\
\hline 2010 & NY State \& Local P & 100 & 2 & A \\
\hline 2010 & Washington LEOFF P & 100 & 2 & A \\
\hline 2010 & Washington PERS 2/ & 100 & 2 & A \\
\hline 2010 & Washington School & 100 & 2 & A \\
\hline 2010 & Washington Teacher & 100 & 2 & A \\
\hline 2010 & Wisconsin Retireme & 99.83667 & 9 & A \\
\hline 2010 & North Carolina Loc & 99.59287 & 10 & A \\
\hline 2010 & Maine Local & 96.34939 & 11 & A- \\
\hline 2010 & South Dakota PERS & 96.30235 & 12 & A- \\
\hline 2010 & Delaware State Emp & 95.95045 & 13 & A- \\
\hline 2010 & North Carolina Tea & 95.36732 & 14 & A- \\
\hline 2010 & Texas County \& Dis & 89.35037 & 15 & A- \\
\hline 2010 & Minnesota State Em & 87.29861 & 16 & A- \\
\hline 2010 & Oregon PERS & 86.94427 & 17 & A- \\
\hline
\end{tabular}




\begin{tabular}{|c|c|c|c|c|}
\hline 2010 & University of Cali & 86.71912 & 18 & A- \\
\hline 2010 & Florida RS & 86.59335 & 19 & A- \\
\hline 2010 & Texas LECOS & 86.26372 & 20 & A- \\
\hline 2010 & Georgia Teachers & 85.74881 & 21 & A- \\
\hline 2010 & Texas ERS & 85.39764 & 22 & $\mathrm{~B}+$ \\
\hline 2010 & Indiana PERF & 85.18651 & 23 & $\mathrm{~B}+$ \\
\hline 2010 & Washington Teacher & 84.67608 & 24 & $\mathrm{~B}+$ \\
\hline 2010 & Wyoming Public Emp & 84.595 & 25 & $\mathrm{~B}+$ \\
\hline 2010 & California PERF & 83.37144 & 26 & $\mathrm{~B}+$ \\
\hline 2010 & Illinois Municipal & 83.25362 & 27 & $\mathrm{~B}+$ \\
\hline 2010 & Texas Teachers & 82.93626 & 28 & $\mathrm{~B}+$ \\
\hline 2010 & Texas Municipal & 82.93338 & 29 & $\mathrm{~B}+$ \\
\hline 2010 & Nebraska Schools & 82.42578 & 30 & $\mathrm{~B}+$ \\
\hline 2010 & Utah Noncontributo & 82.235 & 31 & $\mathrm{~B}+$ \\
\hline 2010 & Iowa PERS & 81.3704 & 32 & B \\
\hline 2010 & Vermont State Empl & 81.15081 & 33 & $\mathrm{~B}$ \\
\hline 2010 & Missouri Local & 81.04596 & 34 & $\mathrm{~B}$ \\
\hline 2010 & Massachusetts SERS & 80.95523 & 35 & B \\
\hline 2010 & Missouri State Emp & 80.41462 & 36 & $\mathrm{~B}$ \\
\hline 2010 & Georgia ERS & 80.06082 & 37 & $\mathrm{~B}$ \\
\hline 2010 & Missouri PEERS & 79.05542 & 38 & $\mathrm{~B}$ \\
\hline 2010 & Idaho PERS & 79.03002 & 39 & $\mathrm{~B}$ \\
\hline 2010 & New Mexico PERF & 78.47799 & 40 & $\mathrm{~B}$ \\
\hline 2010 & Minnesota Teachers & 78.45047 & 41 & B \\
\hline 2010 & Missouri Teachers & 77.70221 & 42 & $\mathrm{~B}$ \\
\hline 2010 & New Jersey Police & 77.05897 & 43 & $\mathrm{~B}-$ \\
\hline 2010 & Arizona SRS & 76.4339 & 44 & B- \\
\hline 2010 & Minnesota PERF & 76.40432 & 45 & B- \\
\hline 2010 & Ohio PERS & 76.10072 & 46 & $\mathrm{~B}-$ \\
\hline 2010 & Pennsylvania State & 75.15123 & 47 & B- \\
\hline 2010 & Pennsylvania Schoo & 75.0668 & 48 & B- \\
\hline 2010 & West Virginia PERS & 74.62891 & 49 & B- \\
\hline 2010 & Michigan Municipal & 74.54393 & 50 & B- \\
\hline 2010 & South Carolina Pol & 74.48164 & 51 & B- \\
\hline 2010 & Montana PERS & 74.20878 & 52 & B- \\
\hline 2010 & Washington PERS 1 & 74.11809 & 53 & B- \\
\hline 2010 & Arkansas PERS & 74.05531 & 54 & $\mathrm{C}+$ \\
\hline 2010 & Arkansas Teachers & 73.76547 & 55 & $\mathrm{C}+$ \\
\hline 2010 & Rhode Island Munic & 73.55031 & 56 & $\mathrm{C}+$ \\
\hline 2010 & North Dakota PERS & 73.43476 & 57 & $\mathrm{C}+$ \\
\hline 2010 & Colorado Municipal & 73.04948 & 58 & $\mathrm{C}+$ \\
\hline 2010 & Ohio School Employ & 72.61528 & 59 & $\mathrm{C}+$ \\
\hline 2010 & Michigan SERS & 72.5573 & 60 & $\mathrm{C}+$ \\
\hline 2010 & Virginia Retiremen & 72.42895 & 61 & $\mathrm{C}+$ \\
\hline 2010 & California Teacher & 71.46219 & 62 & $\mathrm{C}+$ \\
\hline 2010 & Nevada Regular Emp & 71.21079 & 63 & $\mathrm{C}+$ \\
\hline 2010 & Alabama Teachers & 71.14176 & 64 & $\mathrm{C}$ \\
\hline 2010 & Michigan Public Sc & 71.05881 & 65 & $\mathrm{C}$ \\
\hline 2010 & North Dakota Teach & 69.84622 & 66 & $\mathrm{C}$ \\
\hline
\end{tabular}




\begin{tabular}{|c|c|c|c|c|}
\hline 2010 & New Jersey PERS & 69.49479 & 67 & $\mathrm{C}$ \\
\hline 2010 & Ohio Police \& Fire & 69.42738 & 68 & $\mathrm{C}$ \\
\hline 2010 & Alabama ERS & 68.18293 & 69 & $\mathrm{C}$ \\
\hline 2010 & Nevada Police Offi & 67.81105 & 70 & $\mathrm{C}$ \\
\hline 2010 & Arizona Public Saf & 67.73081 & 71 & $\mathrm{C}$ \\
\hline 2010 & New Jersey Teacher & 67.14388 & 72 & $\mathrm{C}$ \\
\hline 2010 & Vermont Teachers & 66.45811 & 73 & $\mathrm{C}$ \\
\hline 2010 & Massachusetts Teac & 66.25765 & 74 & $\mathrm{C}$ \\
\hline 2010 & Maine State and $\mathrm{Te}$ & 66.02651 & 75 & $\mathrm{C}-$ \\
\hline 2010 & Oklahoma PERS & 65.97383 & 76 & $\mathrm{C}-$ \\
\hline 2010 & New Mexico Teacher & 65.70732 & 77 & C- \\
\hline 2010 & Kentucky County & 65.54854 & 78 & $\mathrm{C}-$ \\
\hline 2010 & South Carolina RS & 65.50862 & 79 & $\mathrm{C}-$ \\
\hline 2010 & Montana Teachers & 65.43765 & 80 & $\mathrm{C}-$ \\
\hline 2010 & Maryland Teachers & 65.41274 & 81 & $\mathrm{C}-$ \\
\hline 2010 & Colorado School & 64.84332 & 82 & $\mathrm{C}-$ \\
\hline 2010 & Mississippi PERS & 64.15106 & 83 & $\mathrm{C}-$ \\
\hline 2010 & Colorado State & 62.84061 & 84 & C- \\
\hline 2010 & Maryland PERS & 62.79893 & 85 & $\mathrm{D}$ \\
\hline 2010 & Alaska PERS & 62.37984 & 86 & $\mathrm{D}$ \\
\hline 2010 & Kansas PERS & 62.18446 & 87 & $\mathrm{D}$ \\
\hline 2010 & Connecticut Teache & 61.41582 & 88 & $\mathrm{D}$ \\
\hline 2010 & Hawaii ERS & 61.38165 & 89 & $\mathrm{D}$ \\
\hline 2010 & Kentucky Teachers & 61.00533 & 90 & $\mathrm{D}$ \\
\hline 2010 & Ohio Teachers & 59.06447 & 91 & $\mathrm{D}$ \\
\hline 2010 & New Hampshire Reti & 58.45296 & 92 & $\mathrm{D}$ \\
\hline 2010 & Louisiana SERS & 57.65642 & 93 & $\mathrm{D}$ \\
\hline 2010 & Louisiana Teachers & 54.3551 & 94 & $\mathrm{D}$ \\
\hline 2010 & Alaska Teachers & 54.26799 & 95 & $\mathrm{D}$ \\
\hline 2010 & Illinois Teachers & 48.43776 & 96 & $\mathrm{~F}$ \\
\hline 2010 & Rhode Island ERS & 48.3819 & 97 & $\mathrm{~F}$ \\
\hline 2010 & Oklahoma Teachers & 47.87994 & 98 & $\mathrm{~F}$ \\
\hline 2010 & West Virginia Teac & 46.53408 & 99 & $\mathrm{~F}$ \\
\hline 2010 & Illinois Universit & 46.36934 & 100 & $\mathrm{~F}$ \\
\hline 2010 & Connecticut SERS & 44.40732 & 101 & $\mathrm{~F}$ \\
\hline 2010 & Indiana Teachers & 44.25355 & 102 & $\mathrm{~F}$ \\
\hline 2010 & Missouri DOT and $\mathrm{H}$ & 42.2185 & 103 & $\mathrm{~F}$ \\
\hline 2010 & Kentucky ERS & 40.30588 & 104 & $\mathrm{~F}$ \\
\hline 2010 & Illinois SERS & 37.39932 & 105 & $\mathrm{~F}$ \\
\hline 2011 & NY State \& Local E & 100 & 1 & $\mathrm{~A}$ \\
\hline 2011 & NY State \& Local P & 100 & 1 & $\mathrm{~A}$ \\
\hline 2011 & South Dakota PERS & 96.38537 & 3 & A \\
\hline 2011 & Delaware State Emp & 93.95691 & 4 & $\mathrm{~A}$ \\
\hline 2011 & Maine Local & 93.46841 & 5 & A \\
\hline 2011 & TN State and Teach & 92.08305 & 6 & A \\
\hline 2011 & Idaho PERS & 90.24934 & 7 & A- \\
\hline 2011 & TN Political Subdi & 89.14514 & 8 & A- \\
\hline 2011 & Florida RS & 86.92971 & 9 & A- \\
\hline 2011 & Texas LECOS & 86.42691 & 10 & A- \\
\hline
\end{tabular}




\begin{tabular}{|c|c|c|c|c|}
\hline 2011 & Minnesota State Em & 86.39723 & 11 & A- \\
\hline 2011 & Missouri Teachers & 85.46991 & 12 & A- \\
\hline 2011 & Missouri PEERS & 85.33277 & 13 & A- \\
\hline 2011 & Texas ERS & 84.50336 & 14 & $\mathrm{~B}+$ \\
\hline 2011 & Texas Teachers & 82.72835 & 15 & $\mathrm{~B}+$ \\
\hline 2011 & University of Cali & 82.49314 & 16 & $\mathrm{~B}+$ \\
\hline 2011 & Wyoming Public Emp & 81.86826 & 17 & $\mathrm{~B}+$ \\
\hline 2011 & Missouri Local & 81.55346 & 18 & $\mathrm{~B}+$ \\
\hline 2011 & Oklahoma PERS & 80.67011 & 19 & $\mathrm{~B}+$ \\
\hline 2011 & Indiana PERF & 80.46984 & 20 & $\mathrm{~B}+$ \\
\hline 2011 & Nebraska Schools & 80.39494 & 21 & $\mathrm{~B}$ \\
\hline 2011 & Maine State and $\mathrm{Te}$ & 80.23863 & 22 & B \\
\hline 2011 & Iowa PERS & 79.89258 & 23 & $\mathrm{~B}$ \\
\hline 2011 & Vermont State Empl & 79.55891 & 24 & $\mathrm{~B}$ \\
\hline 2011 & Missouri State Emp & 79.24578 & 25 & B \\
\hline 2011 & West Virginia PERS & 78.37662 & 26 & $\mathrm{~B}$ \\
\hline 2011 & Minnesota Teachers & 77.27212 & 27 & B- \\
\hline 2011 & Georgia ERS & 76.04988 & 28 & B- \\
\hline 2011 & Arizona SRS & 75.52833 & 29 & B- \\
\hline 2011 & Minnesota PERF & 75.17664 & 30 & B- \\
\hline 2011 & New Jersey Police & 74.88585 & 31 & B- \\
\hline 2011 & Arkansas Teachers & 71.81238 & 32 & B- \\
\hline 2011 & Arkansas PERS & 70.68787 & 33 & B- \\
\hline 2011 & Nevada Regular Emp & 70.62969 & 34 & $\mathrm{C}+$ \\
\hline 2011 & North Dakota PERS & 70.53766 & 35 & $\mathrm{C}+$ \\
\hline 2011 & New Mexico PERF & 70.45609 & 36 & $\mathrm{C}+$ \\
\hline 2011 & Montana PERS & 70.24728 & 37 & $\mathrm{C}+$ \\
\hline 2011 & Virginia Retiremen & 69.90645 & 38 & $\mathrm{C}+$ \\
\hline 2011 & Pennsylvania Schoo & 69.05753 & 39 & $\mathrm{C}+$ \\
\hline 2011 & Nevada Police Offi & 68.41859 & 40 & $\mathrm{C}+$ \\
\hline 2011 & New Jersey PERS & 66.77933 & 41 & $\mathrm{C}$ \\
\hline 2011 & Maryland Teachers & 66.29916 & 42 & $\mathrm{C}$ \\
\hline 2011 & North Dakota Teach & 66.28237 & 43 & $\mathrm{C}$ \\
\hline 2011 & Ohio School Employ & 65.21357 & 44 & $\mathrm{C}$ \\
\hline 2011 & Vermont Teachers & 63.75736 & 45 & $\mathrm{C}$ \\
\hline 2011 & Arizona Public Saf & 63.73059 & 46 & $\mathrm{C}$ \\
\hline 2011 & New Jersey Teacher & 63.17063 & 47 & $\mathrm{C}-$ \\
\hline 2011 & New Mexico Teacher & 63.04935 & 48 & $\mathrm{C}-$ \\
\hline 2011 & Kentucky County & 62.91141 & 49 & $\mathrm{C}-$ \\
\hline 2011 & Maryland PERS & 62.8113 & 50 & $\mathrm{C}-$ \\
\hline 2011 & Mississippi PERS & 62.21252 & 51 & $\mathrm{C}-$ \\
\hline 2011 & Montana Teachers & 61.53136 & 52 & $\mathrm{C}-$ \\
\hline 2011 & Hawaii ERS & 59.42608 & 53 & $\mathrm{C}-$ \\
\hline 2011 & Ohio Teachers & 58.83642 & 54 & $\mathrm{D}$ \\
\hline 2011 & Louisiana SERS & 57.57223 & 55 & $\mathrm{D}$ \\
\hline 2011 & New Hampshire Reti & 57.4152 & 56 & $\mathrm{D}$ \\
\hline 2011 & Kentucky Teachers & 57.40812 & 57 & $\mathrm{D}$ \\
\hline 2011 & Oklahoma Teachers & 56.72065 & 58 & $\mathrm{D}$ \\
\hline 2011 & Louisiana Teachers & 55.13728 & 59 & $\mathrm{D}$ \\
\hline
\end{tabular}




\begin{tabular}{|l|l|l|l|l|}
\hline 2011 & West Virginia Teac & 53.72774 & 60 & D \\
\hline 2011 & Illinois Teachers & 46.45741 & 61 & F \\
\hline 2011 & Illinois Universit & 44.25186 & 62 & F \\
\hline 2011 & Indiana Teachers & 43.77038 & 63 & F \\
\hline 2011 & Missouri DOT and H & 43.28285 & 64 & F \\
\hline 2011 & Kentucky ERS & 35.60094 & 65 & F \\
\hline 2011 & Illinois SERS & 35.54653 & 66 & F \\
\hline
\end{tabular}

A snap shot of the complete rank of all pension plans from fiscal year 2001 to 2011, Table 4 shows the ranking and rating for pension plans in 2010 and 2011 only. Actuarial funding ratio in column (3) is calculated as in Eq. (1). "Ranking" in column (4) is a simple rank (descending) of actuarial funding ratio. In column (5) We assign each pension plan a letter rating based on rating structure denoted in Table 2, with A denoting the healthiest pension plans and $\mathrm{F}$ the worst plans.

Six pension plans have the highest A ratings in fiscal year 2011: (1) NY State \& Local Employee, (2) NY State \& Local Police and Firefighters, (3) South Dakota PERS, (4) Delaware State Employees, (5) Maine Local Employees, and (6) TN State and Teachers. Six pension plans have the lowest F ratings in fiscal year 2011: (1) Illinois Teachers, (2) Illinois Universities, (3) Indiana Teachers, (4) Missouri DOT and Highway, (5) Kentucky ERS, and (6) Illinois SERS.

\subsubsection{Ranking Pension Plans by Risky Asset Allocation (Equities + Alternatives)}

Table 5. Ranking Pension Plans by Risky Asset Allocation

\begin{tabular}{|c|c|c|c|c|}
\hline Fiscal Year & Pension Name & Risky Asset Allocation (\%) & Ranking & Letter Rating \\
\hline 2010 & Nebraska Schools & 26.3814 & 1 & $\mathrm{~A}$ \\
\hline 2010 & Texas Municipal & 33.1 & 2 & A \\
\hline 2010 & Washington LEOFF P & 34.71 & 6 & $\mathrm{~A}$ \\
\hline 2010 & Washington Teacher & 34.71 & 6 & $\mathrm{~A}$ \\
\hline 2010 & Washington LEOFF P & 34.71 & 6 & $\mathrm{~A}$ \\
\hline 2010 & Washington PERS $2 /$ & 34.71 & 6 & $\mathrm{~A}$ \\
\hline 2010 & Washington School & 34.71 & 6 & $\mathrm{~A}$ \\
\hline 2010 & Washington Teacher & 34.71 & 6 & A \\
\hline 2010 & Washington PERS 1 & 34.71 & 6 & A \\
\hline 2010 & Vermont State Empl & 37 & 10.5 & $\mathrm{~A}$ \\
\hline 2010 & Vermont Teachers & 37 & 10.5 & A \\
\hline 2010 & Oregon PERS & 37.4 & 12 & A- \\
\hline 2010 & Iowa PERS & 38.67 & 13 & A- \\
\hline 2010 & Arizona Public Saf & 40.95 & 14 & A- \\
\hline 2010 & Missouri DOT and $\mathrm{H}$ & 41.6 & 15 & A- \\
\hline 2010 & Utah Noncontributo & 44.6 & 16 & A- \\
\hline 2010 & TN Political Subdi & 46 & 17.5 & A- \\
\hline 2010 & TN State and Teach & 46 & 17.5 & A- \\
\hline 2010 & Indiana Teachers & 47.6 & 19 & A- \\
\hline 2010 & Virginia Retiremen & 48 & 20 & A- \\
\hline 2010 & Pennsylvania Schoo & 48.8 & 21 & A- \\
\hline 2010 & North Dakota PERS & 49.8 & 22 & $\mathrm{~B}+$ \\
\hline 2010 & Montana Teachers & 51 & 23 & $\mathrm{~B}+$ \\
\hline 2010 & Illinois Teachers & 51.8 & 24 & $\mathrm{~B}+$ \\
\hline 2010 & South Dakota PERS & 52.2 & 25 & $\mathrm{~B}+$ \\
\hline 2010 & Wisconsin Retireme & 52.23692 & 26 & $\mathrm{~B}+$ \\
\hline 2010 & Kansas PERS & 52.38 & 27 & $\mathrm{~B}+$ \\
\hline 2010 & Massachusetts SERS & 52.51 & 28.5 & $\mathrm{~B}+$ \\
\hline 2010 & Massachusetts Teac & 52.51 & 28.5 & $\mathrm{~B}+$ \\
\hline 2010 & South Carolina RS & 53.05 & 30 & $\mathrm{~B}+$ \\
\hline 2010 & South Carolina Pol & 53.22 & 31 & $\mathrm{~B}+$ \\
\hline
\end{tabular}




\begin{tabular}{|c|c|c|c|c|}
\hline 2010 & Nevada Regular Emp & 53.8 & 32.5 & B \\
\hline 2010 & Nevada Police Offi & 53.8 & 32.5 & $\mathrm{~B}$ \\
\hline 2010 & University of Cali & 54.5 & 34 & $\mathrm{~B}$ \\
\hline 2010 & Connecticut Teache & 54.6 & 35 & $\mathrm{~B}$ \\
\hline 2010 & Ohio School Employ & 55 & 36 & $\mathrm{~B}$ \\
\hline 2010 & Alaska PERS & 55.03 & 37 & $\mathrm{~B}$ \\
\hline 2010 & Alaska Teachers & 55.13 & 38 & $\mathrm{~B}$ \\
\hline 2010 & North Carolina Tea & 55.4 & 39.5 & $\mathrm{~B}$ \\
\hline 2010 & North Carolina Loc & 55.4 & 39.5 & $\mathrm{~B}$ \\
\hline 2010 & Idaho PERS & 55.9 & 42 & $\mathrm{~B}$ \\
\hline 2010 & Missouri PEERS & 55.9 & 42 & $\mathrm{~B}$ \\
\hline 2010 & Missouri Teachers & 55.9 & 42 & $\mathrm{~B}$ \\
\hline 2010 & Texas Teachers & 55.9 & 44 & B- \\
\hline 2010 & Hawaii ERS & 56.2 & 45.5 & B- \\
\hline 2010 & New York State Tea & 56.2 & 45.5 & $\mathrm{~B}-$ \\
\hline 2010 & Florida RS & 56.6 & 47 & B- \\
\hline 2010 & Missouri State Emp & 56.7 & 48 & $\mathrm{~B}-$ \\
\hline 2010 & Ohio PERS & 56.95 & 49 & B- \\
\hline 2010 & NY State \& Local E & 57.8 & 50.5 & B- \\
\hline 2010 & NY State \& Local P & 57.8 & 50.5 & B- \\
\hline 2010 & New Jersey PERS & 58.4 & 52 & B- \\
\hline 2010 & Maine State and Te & 58.6 & 53.5 & $\mathrm{C}+$ \\
\hline 2010 & Maine Local & 58.6 & 53.5 & $\mathrm{C}+$ \\
\hline 2010 & Alabama ERS & 58.64 & 55 & $\mathrm{C}+$ \\
\hline 2010 & New Mexico Teacher & 59 & 56 & $\mathrm{C}+$ \\
\hline 2010 & California PERF & 59.21 & 57 & $\mathrm{C}+$ \\
\hline 2010 & Ohio Teachers & 59.47 & 58 & $\mathrm{C}+$ \\
\hline 2010 & Alabama Teachers & 59.7 & 59.5 & $\mathrm{C}+$ \\
\hline 2010 & Kentucky Teachers & 59.7 & 59.5 & $\mathrm{C}+$ \\
\hline 2010 & Illinois SERS & 59.72 & 61 & $\mathrm{C}+$ \\
\hline 2010 & New Jersey Teacher & 60.2 & 62 & $\mathrm{C}+$ \\
\hline 2010 & Arkansas Teachers & 60.3 & 63 & $\mathrm{C}+$ \\
\hline 2010 & Rhode Island Munic & 60.6 & 64.5 & $\mathrm{C}$ \\
\hline 2010 & Rhode Island ERS & 60.6 & 64.5 & $\mathrm{C}$ \\
\hline 2010 & Oklahoma Teachers & 60.75 & 66 & $\mathrm{C}$ \\
\hline 2010 & North Dakota Teach & 61 & 67 & $\mathrm{C}$ \\
\hline 2010 & Oklahoma PERS & 61.1 & 68 & $\mathrm{C}$ \\
\hline 2010 & New Jersey Police & 61.5 & 69 & $\mathrm{C}$ \\
\hline 2010 & Georgia Teachers & 61.9 & 70 & $\mathrm{C}$ \\
\hline 2010 & Texas LECOS & 63.19 & 71.5 & $\mathrm{C}$ \\
\hline 2010 & Texas ERS & 63.19 & 71.5 & $\mathrm{C}$ \\
\hline 2010 & Montana PERS & 63.49 & 73 & $\mathrm{C}$ \\
\hline 2010 & Ohio Police \& Fire & 63.88 & 74 & $\mathrm{C}$ \\
\hline 2010 & New Hampshire Reti & 64.1 & 75 & $\mathrm{C}-$ \\
\hline 2010 & Georgia ERS & 64.2 & 76.5 & $\mathrm{C}-$ \\
\hline 2010 & Indiana PERF & 64.2 & 76.5 & $\mathrm{C}-$ \\
\hline 2010 & Arkansas PERS & 64.8 & 78 & $\mathrm{C}-$ \\
\hline 2010 & Arizona SRS & 65.29 & 79 & $\mathrm{C}-$ \\
\hline 2010 & Kentucky ERS & 65.4 & 80.5 & $\mathrm{C}-$ \\
\hline 2010 & Kentucky County & 65.4 & 80.5 & $\mathrm{C}-$ \\
\hline
\end{tabular}




\begin{tabular}{|c|c|c|c|c|}
\hline 2010 & Illinois Municipal & 65.7 & 82 & $\mathrm{C}-$ \\
\hline 2010 & Maryland PERS & 65.9 & 83.5 & C- \\
\hline 2010 & Maryland Teachers & 65.9 & 83.5 & $\mathrm{C}-$ \\
\hline 2010 & Illinois Universit & 66.3 & 85 & $\mathrm{D}$ \\
\hline 2010 & California Teacher & 66.34 & 86 & $\mathrm{D}$ \\
\hline 2010 & Texas County \& Dis & 66.8 & 87 & $\mathrm{D}$ \\
\hline 2010 & Colorado State & 67 & 89 & $\mathrm{D}$ \\
\hline 2010 & Colorado School & 67 & 89 & $\mathrm{D}$ \\
\hline 2010 & Colorado Municipal & 67 & 89 & $\mathrm{D}$ \\
\hline 2010 & Missouri Local & 67.61171 & 91 & $\mathrm{D}$ \\
\hline 2010 & Wyoming Public Emp & 67.62 & 92 & $\mathrm{D}$ \\
\hline 2010 & West Virginia Teac & 68.02591 & 93 & $\mathrm{D}$ \\
\hline 2010 & Pennsylvania State & 68.8 & 94 & $\mathrm{D}$ \\
\hline 2010 & West Virginia PERS & 68.95282 & 95 & $\mathrm{D}$ \\
\hline 2010 & Mississippi PERS & 69.3 & 96 & $\mathrm{~F}$ \\
\hline 2010 & Delaware State Emp & 70.2 & 97 & $\mathrm{~F}$ \\
\hline 2010 & Louisiana Teachers & 72.898 & 98 & $\mathrm{~F}$ \\
\hline 2010 & Michigan SERS & 72.9 & 99 & $\mathrm{~F}$ \\
\hline 2010 & Minnesota PERF & 73.1 & 100 & $\mathrm{~F}$ \\
\hline 2010 & Minnesota Teachers & 73.15422 & 101 & $\mathrm{~F}$ \\
\hline 2010 & Minnesota State Em & 73.24363 & 102 & $\mathrm{~F}$ \\
\hline 2010 & Louisiana SERS & 73.4 & 103 & $\mathrm{~F}$ \\
\hline 2010 & Michigan Public Sc & 73.9 & 104 & $\mathrm{~F}$ \\
\hline 2010 & New Mexico PERF & 79.6 & 105 & $\mathrm{~F}$ \\
\hline 2011 & Nebraska Schools & 21.7827 & 1 & $\mathrm{~A}$ \\
\hline 2011 & Missouri DOT and $\mathrm{H}$ & 31.5 & 2 & $\mathrm{~A}$ \\
\hline 2011 & Arizona Public Saf & 35.9 & 3 & $\mathrm{~A}$ \\
\hline 2011 & Washington LEOFF 1 & 36.83 & 7 & $\mathrm{~A}$ \\
\hline 2011 & Washington LEOFF 2 & 36.83 & 7 & $\mathrm{~A}$ \\
\hline 2011 & Washington PERS 1 & 36.83 & 7 & $\mathrm{~A}$ \\
\hline 2011 & Washington PERS 2 & 36.83 & 7 & $\mathrm{~A}$ \\
\hline 2011 & Washington School & 36.83 & 7 & $\mathrm{~A}$ \\
\hline 2011 & Washington Teacher 1 & 36.83 & 7 & $\mathrm{~A}$ \\
\hline 2011 & Washington Teacher 2 & 36.83 & 7 & $\mathrm{~A}$ \\
\hline 2011 & Vermont Teachers & 37 & 11.5 & A- \\
\hline 2011 & Vermont State Empl & 37 & 11.5 & A- \\
\hline 2011 & Iowa PERS & 42.48 & 13 & A- \\
\hline 2011 & Pennsylvania Schoo & 47.4 & 14 & A- \\
\hline 2011 & Virginia Retiremen & 48 & 15 & A- \\
\hline 2011 & Ohio School Employ & 50.9 & 16 & A- \\
\hline 2011 & South Dakota PERS & 51.1 & 17 & $\mathrm{~B}+$ \\
\hline 2011 & Illinois Teachers & 51.2 & 18 & $\mathrm{~B}+$ \\
\hline 2011 & TN Political Subdi & 52 & 19.5 & $\mathrm{~B}+$ \\
\hline 2011 & TN State and Teach & 52 & 19.5 & $\mathrm{~B}+$ \\
\hline 2011 & Indiana Teachers & 53.3 & 21 & $\mathrm{~B}+$ \\
\hline 2011 & University of Cali & 53.97 & 22 & $\mathrm{~B}+$ \\
\hline 2011 & Texas Teachers & 54.7 & 23 & $\mathrm{~B}+$ \\
\hline 2011 & Montana Teachers & 55 & 24.5 & $\mathrm{~B}+$ \\
\hline 2011 & New Mexico PERF & 55 & 24.5 & $\mathrm{~B}+$ \\
\hline 2011 & Wisconsin Retireme & 55.03444 & 26 & $\mathrm{~B}$ \\
\hline
\end{tabular}




\begin{tabular}{|c|c|c|c|c|}
\hline 2011 & North Dakota PERS & 55.2 & 27 & $\mathrm{~B}$ \\
\hline 2011 & Massachusetts SERS & 55.4 & 28.5 & $\mathrm{~B}$ \\
\hline 2011 & Massachusetts Teac & 55.4 & 28.5 & $\mathrm{~B}$ \\
\hline 2011 & Missouri State Emp & 55.7 & 30 & $\mathrm{~B}$ \\
\hline 2011 & Alabama ERS & 56 & 31 & $\mathrm{~B}$ \\
\hline 2011 & North Carolina Loc & 56.6 & 32.5 & $\mathrm{~B}$ \\
\hline 2011 & North Carolina Tea & 56.6 & 32.5 & $\mathrm{~B}$ \\
\hline 2011 & Kentucky ERS & 57 & 34.5 & B- \\
\hline 2011 & Kentucky County & 57 & 34.5 & B- \\
\hline 2011 & NY State \& Local E & 57.3 & 36.5 & $\mathrm{~B}-$ \\
\hline 2011 & NY State \& Local P & 57.3 & 36.5 & $\mathrm{~B}-$ \\
\hline 2011 & Alabama Teachers & 57.42 & 38 & B- \\
\hline 2011 & New Mexico Teacher & 58 & 39 & $\mathrm{~B}-$ \\
\hline 2011 & Alaska PERS & 58.25 & 40 & B- \\
\hline 2011 & Alaska Teachers & 58.46 & 41 & B- \\
\hline 2011 & New York State Tea & 58.5 & 42 & $\mathrm{C}+$ \\
\hline 2011 & Nevada Police Offi & 59 & 43.5 & $\mathrm{C}+$ \\
\hline 2011 & Nevada Regular Emp & 59 & 43.5 & $\mathrm{C}+$ \\
\hline 2011 & Idaho PERS & 59.4 & 45 & $\mathrm{C}+$ \\
\hline 2011 & Oklahoma Teachers & 60 & 46 & $\mathrm{C}+$ \\
\hline 2011 & Kansas PERS & 60.65 & 47 & $\mathrm{C}+$ \\
\hline 2011 & Maryland Teachers & 61.8 & 48.5 & $\mathrm{C}+$ \\
\hline 2011 & Maryland PERS & 61.8 & 48.5 & $\mathrm{C}+$ \\
\hline 2011 & Maine Local & 62.3 & 50.5 & $\mathrm{C}$ \\
\hline 2011 & Maine State and Te & 62.3 & 50.5 & $\mathrm{C}$ \\
\hline 2011 & Missouri Teachers & 62.5 & 52.5 & $\mathrm{C}$ \\
\hline 2011 & Missouri PEERS & 62.5 & 52.5 & $\mathrm{C}$ \\
\hline 2011 & South Carolina Pol & 62.69 & 54 & $\mathrm{C}$ \\
\hline 2011 & South Carolina RS & 62.83 & 55 & $\mathrm{C}$ \\
\hline 2011 & California PERF & 63.13 & 56 & $\mathrm{C}$ \\
\hline 2011 & Arizona SRS & 63.29 & 57 & $\mathrm{C}$ \\
\hline 2011 & Florida RS & 63.4 & 58 & $\mathrm{C}$ \\
\hline 2011 & Kentucky Teachers & 63.5 & 59 & $\mathrm{C}-$ \\
\hline 2011 & Ohio Teachers & 63.66 & 60 & $\mathrm{C}-$ \\
\hline 2011 & Hawaii ERS & 64.4 & 61 & $\mathrm{C}-$ \\
\hline 2011 & Texas ERS & 64.63 & 62.5 & $\mathrm{C}-$ \\
\hline 2011 & Texas LECOS & 64.63 & 62.5 & $\mathrm{C}-$ \\
\hline 2011 & North Dakota Teach & 65 & 64 & $\mathrm{C}-$ \\
\hline 2011 & New Hampshire Reti & 66.6 & 65 & $\mathrm{C}-$ \\
\hline 2011 & Oklahoma PERS & 67 & 66 & $\mathrm{C}-$ \\
\hline 2011 & West Virginia Teac & 67.10659 & 67 & $\mathrm{D}$ \\
\hline 2011 & Montana PERS & 67.33 & 68 & $\mathrm{D}$ \\
\hline 2011 & California Teacher & 67.8 & 69 & $\mathrm{D}$ \\
\hline 2011 & Indiana PERF & 68.5 & 70 & $\mathrm{D}$ \\
\hline 2011 & Michigan SERS & 69.2 & 71 & $\mathrm{D}$ \\
\hline 2011 & West Virginia PERS & 69.2606 & 72 & $\mathrm{D}$ \\
\hline 2011 & Michigan Public Sc & 69.9 & 73 & $\mathrm{D}$ \\
\hline 2011 & Louisiana SERS & 70 & 74 & $\mathrm{D}$ \\
\hline 2011 & Mississippi PERS & 71 & 75 & $\mathrm{~F}$ \\
\hline 2011 & Georgia Teachers & 72.3 & 76 & $\mathrm{~F}$ \\
\hline
\end{tabular}




\begin{tabular}{|l|l|l|l|l|}
\hline 2011 & Delaware State Emp & 72.6 & 77 & $\mathrm{~F}$ \\
\hline 2011 & Georgia ERS & 73.8 & 78 & $\mathrm{~F}$ \\
\hline 2011 & Louisiana Teachers & 74.343 & 79 & $\mathrm{~F}$ \\
\hline 2011 & Minnesota Teachers & 75.19407 & 80 & $\mathrm{~F}$ \\
\hline 2011 & Minnesota PERF & 75.4 & 81 & $\mathrm{~F}$ \\
\hline 2011 & Missouri Local & 77.58282 & 82 & $\mathrm{~F}$ \\
\hline
\end{tabular}

Table 5 shows the ranking and rating for pension plans in fiscal year 2010 and 2011 based on the risky asset allocation percentage. "Ranking" in column (4) is a simple rank (ascending) of risky asset allocation. In column (5) we assign each pension plan a letter rating based on rating structure denoted in Table 2, with A denoting the safest pension plans and $\mathrm{F}$ the riskiest plans.

Pension plans have the highest A ratings (or safest pension plans) in fiscal year 2011 are as follows: (1) Nebraska Schools, (2) Missouri DOT and Highway, (3) Arizona Public Safety, and (4) several Washington state pension plans. The following pension plans are rated as "riskiest": (1) Mississippi PERS, (2) Georgia Teachers, (3) Delaware State Employees, (4) Georgia ERS, (5) Louisiana Teachers, (6) Minnesota Teachers, (7) Minnesota PERF, and (8) Missouri Local Employees.

\subsubsection{Ranking Pension Plans by a Combination of Variables}

We now use a combination of 10 variables to rate pension plans, including (1) Actuarial Funding Ratio, (2) Risky Asset Allocation \%, (3) \% of Annual Required Contribution Paid, (4) 1-Year Actual Return of Pension Assets, (5) Actuarial rate (Pension Liab. Discount Rate), (6) Projected Total Annual Required Contribution as a \% of Payroll, (7) Total Normal Cost as a \% of Payroll, (8) Inflation Rate Assumption, (9) Active to Retired Employee Ratio, and (10) \% of Unionized Employees. We here use both a simple ranking and a PCA method.

\section{A. A Simple Ranking Method}

With a simple ranking method, We rank each of the 10 variables (mentioned in the above section) into 10 groups from the best to the worst, and then We sum all the ranks for these 10 variables -this is called "Aggregate Rank", which ranges from 1 to $100(10$ variables $\times 10$ ranks $=100)$. We sort this "Aggregate Rank" into 10 groups and assign the letter rating (as shown in the rating structure of Table 2), with A denoting the best pension plans and $\mathrm{F}$ the worst plans.

Table 6. Ranking Pension Plans based on Multivariable Variables: A Simple Ranking Method

\begin{tabular}{|l|l|l|l|}
\hline Fiscal Year & Pension Name & Aggregate Rank & Letter Rating \\
\hline 2010 & Georgia Teachers & 70 & A \\
\hline 2010 & Indiana Teachers & 70 & A \\
\hline 2010 & Iowa PERS & 70 & A \\
\hline 2010 & North Carolina Tea & 74 & A \\
\hline 2010 & Oregon PERS & 74 & A \\
\hline 2010 & Virginia Retiremen & 74 & A \\
\hline 2010 & Texas Teachers & 75 & A \\
\hline 2010 & Missouri PEERS & 66 & A- \\
\hline 2010 & Missouri State Emp & 66 & A- \\
\hline 2010 & Texas LECOS & 66 & A- \\
\hline 2010 & Nebraska Schools & 67 & A- \\
\hline 2010 & Washington PERS 2/ & 67 & A- \\
\hline 2010 & Washington School & 67 & A- \\
\hline 2010 & South Carolina Pol & 68 & A- \\
\hline 2010 & South Carolina RS & 68 & A- \\
\hline 2010 & Alabama ERS & 55 & B \\
\hline 2010 & Arizona SRS & 57 & B \\
\hline 2010 & New Mexico Teacher & 57 & B \\
\hline 2010 & Wyoming Public Emp & 57 & B \\
\hline 2010 & Minnesota PERF & 59 & B \\
\hline 2010 & Arkansas Teachers & 60 & B \\
\hline & & & \\
\hline
\end{tabular}




\begin{tabular}{|c|c|c|c|}
\hline 2010 & Mississippi PERS & 60 & $\mathrm{~B}$ \\
\hline 2010 & North Dakota PERS & 60 & $\mathrm{~B}$ \\
\hline 2010 & Arizona Public Saf & 52 & B- \\
\hline 2010 & Arkansas PERS & 52 & B- \\
\hline 2010 & Ohio School Employ & 52 & B- \\
\hline 2010 & Oklahoma PERS & 52 & B- \\
\hline 2010 & Montana Teachers & 53 & B- \\
\hline 2010 & West Virginia Teac & 53 & B- \\
\hline 2010 & Illinois Municipal & 54 & B- \\
\hline 2010 & North Dakota Teach & 54 & B- \\
\hline 2010 & Oklahoma Teachers & 54 & B- \\
\hline 2010 & Rhode Island Munic & 54 & B- \\
\hline 2010 & Delaware State Emp & 62 & $\mathrm{~B}+$ \\
\hline 2010 & Kansas PERS & 62 & $\mathrm{~B}+$ \\
\hline 2010 & Kentucky County & 62 & $\mathrm{~B}+$ \\
\hline 2010 & West Virginia PERS & 62 & $\mathrm{~B}+$ \\
\hline 2010 & Colorado Municipal & 63 & $\mathrm{~B}+$ \\
\hline 2010 & Idaho PERS & 63 & $\mathrm{~B}+$ \\
\hline 2010 & Washington LEOFF P & 65 & $\mathrm{~B}+$ \\
\hline 2010 & Washington Teacher & 65 & $\mathrm{~B}+$ \\
\hline 2010 & Louisiana SERS & 47 & $\mathrm{C}$ \\
\hline 2010 & New Mexico PERF & 47 & $\mathrm{C}$ \\
\hline 2010 & Rhode Island ERS & 47 & $\mathrm{C}$ \\
\hline 2010 & Colorado School & 48 & $\mathrm{C}$ \\
\hline 2010 & Pennsylvania Schoo & 48 & $\mathrm{C}$ \\
\hline 2010 & Texas ERS & 48 & $\mathrm{C}$ \\
\hline 2010 & Missouri Teachers & 49 & $\mathrm{C}$ \\
\hline 2010 & Nevada Regular Emp & 49 & $\mathrm{C}$ \\
\hline 2010 & Washington PERS 1 & 49 & $\mathrm{C}$ \\
\hline 2010 & Washington Teacher & 49 & $\mathrm{C}$ \\
\hline 2010 & Colorado State & 44 & C- \\
\hline 2010 & Kentucky Teachers & 44 & C- \\
\hline 2010 & Maryland PERS & 44 & $\mathrm{C}-$ \\
\hline 2010 & Minnesota Teachers & 44 & C- \\
\hline 2010 & Illinois Universit & 45 & $\mathrm{C}-$ \\
\hline 2010 & Kentucky ERS & 45 & $\mathrm{C}-$ \\
\hline 2010 & New Hampshire Reti & 46 & C- \\
\hline 2010 & Alabama Teachers & 50 & $\mathrm{C}+$ \\
\hline 2010 & New Jersey PERS & 50 & $\mathrm{C}+$ \\
\hline 2010 & Hawaii ERS & 51 & $\mathrm{C}+$ \\
\hline 2010 & Maryland Teachers & 51 & $\mathrm{C}+$ \\
\hline 2010 & Montana PERS & 51 & $\mathrm{C}+$ \\
\hline 2010 & Ohio PERS & 51 & $\mathrm{C}+$ \\
\hline 2010 & California Teacher & 40 & $\mathrm{D}$ \\
\hline 2010 & Connecticut Teache & 40 & $\mathrm{D}$ \\
\hline 2010 & Alaska PERS & 41 & $\mathrm{D}$ \\
\hline 2010 & Ohio Teachers & 42 & $\mathrm{D}$ \\
\hline 2010 & Pennsylvania State & 42 & $\mathrm{D}$ \\
\hline 2010 & Nevada Police Offi & 43 & $\mathrm{D}$ \\
\hline
\end{tabular}




\begin{tabular}{|c|c|c|c|}
\hline 2010 & New Jersey Teacher & 43 & $\mathrm{D}$ \\
\hline 2010 & Ohio Police \& Fire & 43 & $\mathrm{D}$ \\
\hline 2010 & Illinois SERS & 31 & $\mathrm{~F}$ \\
\hline 2010 & Illinois Teachers & 35 & $\mathrm{~F}$ \\
\hline 2010 & Alaska Teachers & 36 & $\mathrm{~F}$ \\
\hline 2010 & New Jersey Police & 36 & $\mathrm{~F}$ \\
\hline 2010 & Louisiana Teachers & 38 & $\mathrm{~F}$ \\
\hline 2010 & Michigan Public Sc & 39 & $\mathrm{~F}$ \\
\hline 2010 & Michigan SERS & 39 & $\mathrm{~F}$ \\
\hline 2011 & Texas Teachers & 69 & A \\
\hline 2011 & Virginia Retiremen & 70 & A \\
\hline 2011 & Missouri PEERS & 74 & $\mathrm{~A}$ \\
\hline 2011 & New Mexico PERF & 66 & A- \\
\hline 2011 & Delaware State Emp & 68 & A- \\
\hline 2011 & Arizona SRS & 61 & $\mathrm{~B}$ \\
\hline 2011 & Indiana Teachers & 61 & $\mathrm{~B}$ \\
\hline 2011 & Iowa PERS & 62 & $\mathrm{~B}$ \\
\hline 2011 & Missouri State Emp & 62 & $\mathrm{~B}$ \\
\hline 2011 & Montana Teachers & 58 & B- \\
\hline 2011 & Nebraska Schools & 58 & B- \\
\hline 2011 & Ohio School Employ & 59 & B- \\
\hline 2011 & Idaho PERS & 63 & $\mathrm{~B}+$ \\
\hline 2011 & Kentucky County & 63 & $\mathrm{~B}+$ \\
\hline 2011 & Minnesota PERF & 63 & $\mathrm{~B}+$ \\
\hline 2011 & North Dakota PERS & 63 & $\mathrm{~B}+$ \\
\hline 2011 & Texas LECOS & 63 & $\mathrm{~B}+$ \\
\hline 2011 & Arizona Public Saf & 52 & $\mathrm{C}$ \\
\hline 2011 & Oklahoma Teachers & 52 & $\mathrm{C}$ \\
\hline 2011 & New Mexico Teacher & 53 & $\mathrm{C}$ \\
\hline 2011 & Louisiana SERS & 47 & C- \\
\hline 2011 & Maryland Teachers & 47 & C- \\
\hline 2011 & Kentucky Teachers & 50 & $\mathrm{C}-$ \\
\hline 2011 & Montana PERS & 51 & C- \\
\hline 2011 & Missouri Teachers & 54 & $\mathrm{C}+$ \\
\hline 2011 & North Dakota Teach & 54 & $\mathrm{C}+$ \\
\hline 2011 & Mississippi PERS & 55 & $\mathrm{C}+$ \\
\hline 2011 & Oklahoma PERS & 56 & $\mathrm{C}+$ \\
\hline 2011 & Pennsylvania Schoo & 45 & $\mathrm{D}$ \\
\hline 2011 & Louisiana Teachers & 46 & $\mathrm{D}$ \\
\hline 2011 & Texas ERS & 46 & $\mathrm{D}$ \\
\hline 2011 & Kentucky ERS & 39 & $\mathrm{~F}$ \\
\hline 2011 & Illinois Teachers & 41 & $\mathrm{~F}$ \\
\hline 2011 & Minnesota Teachers & 44 & $\mathrm{~F}$ \\
\hline
\end{tabular}

Table 6 shows the "Aggregate Rank" and rating for pension plans in fiscal year 2010 and 2011 based on these 10 variables. Five pension plans have the highest A or A- ratings in fiscal year 2011: (1) Texas Teachers, (2) Virginia Retirement, (3) Missouri PEERS, (4) New Mexico PERF, and (5) Delaware State Employees. Five pension plans have the lowest F or D ratings in fiscal year 2011: (1) Pennsylvania School, (2) Louisiana Teachers, (3) Texas ERS, (4) Kentucky ERS, and (5) Illinois Teachers. This rating framework is more comprehensive and accurate than previous method which only uses one single rating variable. It paints a colorful picture of the US public pension funds status. 
The rationale is that pension plans have a number of important dimensions while only using pension actuarial ratio or risky asset allocation to rank them might not be an optimal way.

\section{B. PCA Method}

Table 7. The Result of PCA Method

\begin{tabular}{|c|c|c|c|c|c|c|}
\hline \multirow[t]{12}{*}{ Panel A: } & & \multicolumn{4}{|c|}{ Eigenvalues of the Covariance Matrix } & \\
\hline & & Eigenvalue & Difference & Proportion & Cumulative & \\
\hline & 1 & 1038.5950 & 734.3147 & 0.6260 & 0.6260 & \\
\hline & 2 & 304.2803 & 135.3629 & 0.1834 & 0.8094 & \\
\hline & 3 & 168.9174 & 86.6442 & 0.1018 & 0.9113 & \\
\hline & 4 & 82.2732 & 27.6112 & 0.0496 & 0.9608 & \\
\hline & 5 & 54.6620 & 46.1511 & 0.0329 & 0.9938 & \\
\hline & 6 & 8.5110 & 6.7456 & 0.0051 & 0.9989 & \\
\hline & 7 & 1.7653 & 1.7397 & 0.0011 & 1.0000 & \\
\hline & 8 & 0.0256 & 0.0256 & 0.0000 & 1.0000 & \\
\hline & 9 & 0.0000 & 0.0000 & 0.0000 & 1.0000 & \\
\hline & 10 & 0.0000 & & 0.0000 & 1.0000 & \\
\hline \multirow[t]{2}{*}{ Panel B: } & & & \multicolumn{2}{|c|}{ Eigenvectors } & & \\
\hline & & Prin1 & Prin2 & Prin3 & Prin4 & Prin5 \\
\hline fundingratio & & 0.1393 & 0.9012 & 0.1470 & 0.0371 & 0.3646 \\
\hline bargain_cov_pct & & -0.0009 & 0.0000 & -0.0007 & 0.0031 & 0.0033 \\
\hline percentarc & & 0.9866 & -0.1586 & 0.0390 & -0.0016 & 0.0008 \\
\hline inflationassump & & 0.0000 & 0.0001 & 0.0000 & 0.0001 & -0.0001 \\
\hline invreturnassump & & 0.0000 & 0.0000 & 0.0000 & 0.0001 & 0.0001 \\
\hline totarc & & -0.0555 & -0.3663 & -0.0604 & 0.0553 & 0.8147 \\
\hline totnc & & -0.0138 & -0.0684 & -0.0173 & 0.0159 & 0.4460 \\
\hline riskyinv & & -0.0195 & -0.0574 & 0.2989 & 0.9499 & -0.0616 \\
\hline ret_1yr & & -0.0602 & -0.1407 & 0.9400 & -0.3041 & 0.0231 \\
\hline actives_retirees & & 0.0039 & 0.0287 & -0.0066 & -0.0218 & 0.0069 \\
\hline \multirow{2}{*}{\multicolumn{2}{|c|}{ Panel C: }} & \multicolumn{3}{|c|}{ Eigenvectors } & & \\
\hline & & Prin6 & Prin7 & Prin8 & Prin9 & Prin10 \\
\hline fundingratio & & -0.1077 & -0.0317 & -0.0015 & 0.0000 & 0.0000 \\
\hline bargain_cov_pct & & 0.0008 & -0.0145 & 0.9999 & 0.0010 & -0.0069 \\
\hline percentarc & & 0.0035 & 0.0011 & 0.0009 & 0.0000 & 0.0000 \\
\hline inflationassump & & 0.0003 & 0.0005 & -0.0017 & 0.9943 & -0.1070 \\
\hline invreturnassump & & 0.0001 & 0.0003 & 0.0068 & 0.1070 & 0.9942 \\
\hline totarc & & -0.4383 & -0.0124 & -0.0028 & 0.0002 & 0.0000 \\
\hline totnc & & 0.8912 & 0.0365 & -0.0018 & -0.0003 & -0.0001 \\
\hline riskyinv & & 0.0140 & 0.0255 & -0.0021 & -0.0001 & -0.0001 \\
\hline ret_1yr & & 0.0002 & 0.0036 & 0.0015 & 0.0000 & 0.0000 \\
\hline actives_retirees & & -0.0418 & 0.9983 & 0.0145 & -0.0005 & -0.0003 \\
\hline
\end{tabular}

Table 7 shows the result of PCA method. It shows the first principal component explains $62.60 \%$ of total variance and the first 7 principal components (each with an Eigenvalue > 1) explain all variance. As the first principal component explains a fairly large proportion of total variance, we use the first principal component in my analysis. 
Table 8. Ranking Pension Plans based on Multivariable Variables: PCA Method

\begin{tabular}{|c|c|c|}
\hline Fiscal Year & Pension Name & Letter Rating \\
\hline 2010 & Arizona Public Saf & $\mathrm{A}$ \\
\hline 2010 & Arkansas Teachers & $\mathrm{A}$ \\
\hline 2010 & Delaware State Emp & $\mathrm{A}$ \\
\hline 2010 & Idaho PERS & $\mathrm{A}$ \\
\hline 2010 & Kentucky County & $\mathrm{A}$ \\
\hline 2010 & North Carolina Tea & A \\
\hline 2010 & Oregon PERS & $\mathrm{A}$ \\
\hline 2010 & Washington LEOFF P & $\mathrm{A}$ \\
\hline 2010 & Arizona SRS & A- \\
\hline 2010 & Arkansas PERS & A- \\
\hline 2010 & Colorado Municipal & A- \\
\hline 2010 & Georgia Teachers & A- \\
\hline 2010 & Hawaii ERS & A- \\
\hline 2010 & Missouri State Emp & A- \\
\hline 2010 & Nebraska Schools & A- \\
\hline 2010 & South Carolina Pol & A- \\
\hline 2010 & Illinois Municipal & $\mathrm{B}$ \\
\hline 2010 & Iowa PERS & $\mathrm{B}$ \\
\hline 2010 & Maryland Teachers & $\mathrm{B}$ \\
\hline 2010 & Missouri PEERS & $\mathrm{B}$ \\
\hline 2010 & Montana Teachers & $\mathrm{B}$ \\
\hline 2010 & Nevada Regular Emp & $\mathrm{B}$ \\
\hline 2010 & New Hampshire Reti & $\mathrm{B}$ \\
\hline 2010 & Rhode Island ERS & B \\
\hline 2010 & Illinois SERS & B- \\
\hline 2010 & Indiana Teachers & $\mathrm{B}-$ \\
\hline 2010 & Michigan SERS & B- \\
\hline 2010 & Nevada Police Offi & B- \\
\hline 2010 & New Mexico PERF & B- \\
\hline 2010 & Texas Teachers & B- \\
\hline 2010 & Washington PERS 2/ & B- \\
\hline 2010 & West Virginia PERS & B- \\
\hline 2010 & Alabama ERS & $\mathrm{B}+$ \\
\hline 2010 & Alabama Teachers & $\mathrm{B}+$ \\
\hline 2010 & Connecticut Teache & $\mathrm{B}+$ \\
\hline 2010 & Mississippi PERS & $\mathrm{B}+$ \\
\hline 2010 & Ohio PERS & $\mathrm{B}+$ \\
\hline 2010 & Ohio School Employ & $\mathrm{B}+$ \\
\hline 2010 & Rhode Island Munic & $\mathrm{B}+$ \\
\hline 2010 & South Carolina RS & $\mathrm{B}+$ \\
\hline 2010 & Alaska Teachers & $\mathrm{C}$ \\
\hline 2010 & Louisiana Teachers & $\mathrm{C}$ \\
\hline 2010 & Minnesota PERF & $\mathrm{C}$ \\
\hline 2010 & North Dakota Teach & $\mathrm{C}$ \\
\hline 2010 & Oklahoma Teachers & $\mathrm{C}$ \\
\hline 2010 & Washington School & $\mathrm{C}$ \\
\hline 2010 & Washington Teacher & $\mathrm{C}$ \\
\hline 2010 & Wyoming Public Emp & $\mathrm{C}$ \\
\hline
\end{tabular}




\begin{tabular}{|c|c|c|}
\hline 2010 & Colorado School & $\mathrm{C}-$ \\
\hline 2010 & Kansas PERS & $\mathrm{C}-$ \\
\hline 2010 & Kentucky Teachers & C- \\
\hline 2010 & Maryland PERS & C- \\
\hline 2010 & New Jersey Police & $\mathrm{C}-$ \\
\hline 2010 & Oklahoma PERS & C- \\
\hline 2010 & Texas ERS & $\mathrm{C}-$ \\
\hline 2010 & Virginia Retiremen & $\mathrm{C}-$ \\
\hline 2010 & Alaska PERS & $\mathrm{C}+$ \\
\hline 2010 & Illinois Teachers & $\mathrm{C}+$ \\
\hline 2010 & Louisiana SERS & $\mathrm{C}+$ \\
\hline 2010 & Michigan Public Sc & $\mathrm{C}+$ \\
\hline 2010 & Missouri Teachers & $\mathrm{C}+$ \\
\hline 2010 & New Mexico Teacher & $\mathrm{C}+$ \\
\hline 2010 & Texas LECOS & $\mathrm{C}+$ \\
\hline 2010 & West Virginia Teac & $\mathrm{C}+$ \\
\hline 2010 & California Teacher & $\mathrm{D}$ \\
\hline 2010 & Colorado State & $\mathrm{D}$ \\
\hline 2010 & Illinois Universit & $\mathrm{D}$ \\
\hline 2010 & Minnesota Teachers & $\mathrm{D}$ \\
\hline 2010 & Montana PERS & $\mathrm{D}$ \\
\hline 2010 & North Dakota PERS & $\mathrm{D}$ \\
\hline 2010 & Ohio Teachers & $\mathrm{D}$ \\
\hline 2010 & Ohio Police \& Fire & $\mathrm{D}$ \\
\hline 2010 & Kentucky ERS & $\mathrm{F}$ \\
\hline 2010 & New Jersey PERS & $\mathrm{F}$ \\
\hline 2010 & New Jersey Teacher & $\mathrm{F}$ \\
\hline 2010 & Pennsylvania State & $\mathrm{F}$ \\
\hline 2010 & Pennsylvania Schoo & $\mathrm{F}$ \\
\hline 2010 & Washington Teacher & $\mathrm{F}$ \\
\hline 2010 & Washington PERS 1 & $\mathrm{~F}$ \\
\hline 2011 & Kentucky Teachers & $\mathrm{A}$ \\
\hline 2011 & Kentucky County & $\mathrm{A}$ \\
\hline 2011 & Minnesota PERF & $\mathrm{A}$ \\
\hline 2011 & Arizona Public Saf & A- \\
\hline 2011 & Delaware State Emp & A- \\
\hline 2011 & Missouri PEERS & A- \\
\hline 2011 & Missouri State Emp & A- \\
\hline 2011 & Missouri Teachers & $\mathrm{B}$ \\
\hline 2011 & Montana Teachers & $\mathrm{B}$ \\
\hline 2011 & Nebraska Schools & $\mathrm{B}$ \\
\hline 2011 & New Mexico PERF & $\mathrm{B}$ \\
\hline 2011 & Idaho PERS & B- \\
\hline 2011 & Louisiana Teachers & B- \\
\hline 2011 & Texas Teachers & $\mathrm{B}-$ \\
\hline 2011 & Arizona SRS & $\mathrm{B}+$ \\
\hline 2011 & Mississippi PERS & $\mathrm{B}+$ \\
\hline 2011 & Ohio School Employ & $\mathrm{B}+$ \\
\hline 2011 & Illinois Teachers & $\mathrm{C}$ \\
\hline 2011 & Maryland Teachers & $\mathrm{C}$ \\
\hline
\end{tabular}




\begin{tabular}{|l|l|l|}
2011 & Oklahoma Teachers & C \\
\hline 2011 & Minnesota Teachers & C- \\
\hline 2011 & North Dakota Teach & C- \\
\hline 2011 & Oklahoma PERS & C- \\
\hline 2011 & Texas LECOS & C- \\
\hline 2011 & Indiana Teachers & C+ \\
\hline 2011 & Iowa PERS & C+ \\
\hline 2011 & Louisiana SERS & C+ \\
\hline 2011 & New Mexico Teacher & C+ \\
\hline 2011 & Kentucky ERS & D \\
\hline 2011 & Montana PERS & D \\
\hline 2011 & Texas ERS & D \\
\hline 2011 & North Dakota PERS & F \\
\hline 2011 & Pennsylvania Schoo & F \\
\hline 2011 & Virginia Retiremen & F \\
\hline
\end{tabular}

Table8 shows the letter ranking of all pension plans (during 2010 and 2011) using PCA method. Seven pension plans have the highest A or A- ratings in fiscal year 2011: (1) Kentucky Teachers, (2) Kentucky County, (3) Minnesota PERF, (4) Arizona Public Safety, (5) Delaware State Employee, (6) Missouri PEERS, and (7) Missouri State Employee. Six pension plans have the lowest F or D ratings in fiscal year 2011: (1) Kentucky ERS, (2) Montana PERS, (3) Texas ERS, (4) North Dakota PERS, (5) Pennsylvania School, and (6) Virginia Retirement. The rating results are consistent with previous results.

\section{Conclusions}

The 50 states across the US are currently facing a big issue with their government-sponsored pension plans. As the first comprehensive study on the current state of public pension plans, this study has important impact on the American people's retirement security. It provides the first rating of all the government pension plans after incorporating a number of important dimensions, including pension funding levels, financial heath, investment risk, state fiscal constraints, and workforce/retirees demographics. We use (1) actuarial funding ratio, (2) risky asset allocation percentage, and (3) a combination of 10 variables as ranking variables to rate all pension plans. These variables include funding ratio, risky asset allocation, $\%$ of annual required contribution paid, 1 -year actual return of pension assets, actuarial discount rate, projected total annual required Contribution as a $\%$ of payroll, total normal cost as a $\%$ of payroll, inflation rate assumption, active to retired employee ratio, and $\%$ of unionized employees. When considering a combination of 10 variables, we use both a simple "aggregate" ranking method and a principal component analysis method.

Our study complements previous studies on the significant issue of the US public pension funds. It has been estimated that the total pension liabilities for the 50 states to be $\$ 2.97$ trillion in 2008 (Novy-Marx and Rauh, 2009) and the "already-promised" benefits between \$3.21 and \$5.2 trillion (Novy-Marx and Rauh, 2010). Rauh (2011) estimates that the latest total pension liabilities to be $\$ 7.03$ trillion, mainly due to the decreased Treasury yields, which is used to discount future pension payments. The pension funding gap has been estimated to be between $\$ 1.27$ and $\$ 3.26$ trillion (Novy-Marx and Rauh, 2009). Similar findings have been reported by several large consulting firms and research institutions that have focus on public pension issues for a long time. Our studyhelps public employees/retirees to understand their pension plans, and to raise the public awareness of the pension issues.

One limitation of this study is that when we use risky asset allocation to rate pension funds, risky asset allocation is the percentage of a pension plan assets invested in equity market and alternatives (i.e., private equity and venture capital). Jin et al. (2006) propose an innovative measure of pension risk, pension asset beta, which is estimated as the weighted average beta of all asset classes in a pensionfund. Future research can use this alternative measure of pension investment risk. In addition, the effects of pension underfunding and investment risk on a state government fiscal policy, tax rate, or municipal bond yield can be other interesting research questions.

\section{Acknowledgements}

In this paper, the research was sponsored by the soft science key project of Zhejiang Province (2014C25032) and Zhejiang Provincial Key Research Base of Management Science and Engineering and Zhejiang Industrial Development Policy Key Research Centre of Philosophy and Social Science of Zhejiang Province. 


\section{References}

Bellandi, D. (2011). Illinois Income Tax Increase: Legislature Approves 66\% Tax Hike, Breaking News and Opinion on The Huffington Post. Jan. 12, 2011.

Burson, J., Carlson, J., Erungor, O., \& Waiwood, P. (2013). Do Public Pension Obligations Affect State Funding Costs? FRB of Cleveland Working Paper No. 13-01. Available at SSRN: http://ssrn.com/abstract=2248212

Butler, A., Fauver, W., \& Mortal, S. (2010). Corruption, political connections, and municipal finance. Review of Financial Studies, 22, 2873-2905.http://dx.doi.org/10.1093/rfs/hhp010

Capeci, J. (1994). Local fiscal policies, default risk, and municipal borrowing cost. Journal of Public Economics, 53, 73-89. http://dx.doi.org/10.1016/0047-2727(94)90014-0

Center on Budget and Priorities. http://www.cbpp.org/cms/index.cfm?fa=media\&mediaType=audio\&year=2012\&numReturn $=50$

Center for Retirement Research at Boston College. (2011). Public Plans Database. Chestnut Hill, MA: Center for Retirement Research at Boston College. http://pubplans.bc.edu/pls/htmldb/f?p=198:3:3667012107971550

Don, B. (2007). No Such Thing As Soft Debt: http://www.sandiegoreader.com/news/2007/sep/27/no-such-thing-as-soft-debt/

Gao, P., \& Qi, Y. (2013). Political Uncertainty and Public Financing Costs: Evidence from U.S. Municipal Bond Market. Working paper, University of Notre Dame and Concordia University. http://dx.doi.org/10.2139/ssrn.1992200

Gore, A. (2004). Does Mandatory Disclosure Reduce the Cost of Capital? Evidence from Bonds (July 12, 2004). http://dx.doi.org/10.2139/ssrn.565182

Green, J. (2011). Pittsburgh Revival Endangered by \$650 Million Pension Shortfall. Bloomberg, March 30, 2011.

Green, R., Li, D., \& Schurhoff, N. (2010). Price Discovery in Illiquid Markets: Do Financial Asset Prices Rise Faster Than They Fall. Journal of Finance, 65, 1669-1702.Available at SSRN: http://ssrn.com/abstract=1102503

Healey, T., Hess, C., \& Nicholson, K. (2012). Underfunded public pension in the United States: The size of the problem, the obstacles to reform and the path forward. Working paper, Harvard Kennedy School.

Jin, L., Merton, R., Bodie, Z. (2006). Do a firm's equity returns reflect the risk of its pension plan? Journal of Financial Economics, 81, 1-26. http://dx.doi.org/10.1016/j.jfineco.2005.06.005

Jorion, P., Liu, Z., Shi, C. (2005). Informational effects of regulation FD: Evidence from rating agencies. Journal of Financial Economics, 76(2), 309-330. http://dx.doi.org/10.1016/j.jfineco.2004.05.001

Maher, K. (2009). Pittsburgh Pushes Tax on College Students: Local Colleges Give Poor Grades to Proposal by Mayor Ravenstahl That Aims to Raise Money to Alleviate City's Pension Woes. The Wall Street Journal, December 1, 2009.

Marlowe, J. (2013). Municipal Bond Liquidity Before and After the Financial Crisis (January 25, 2013). http://dx.doi.org/10.2139/ssrn.2206730

Merton, R. (2006a). CFOs: Don't forget pension funds. Interview transcript, interview by J. Chernoff, Pensions and Investments, December 11, 2006.

Mohan, N., Zhang, T. (2012). An Analysis of Risk-Taking Behavior for Public Defined Benefit Pension Plans, Upjohn Institute Working Paper No. 12-179.

Mohan, N., Zhang, T. (2014). An Analysis of Risk-Taking Behavior for Public Defined Benefit Pension Plans, Journal of Banking and Finance, 40, 403-419.http://dx.doi.org/10.1016/j.jbankfin.2013.12.011

Novy-Marx, R., \& Rauh, J. (2010). The Crisis in Local Government Pensions in the United States. Brookings-Nomura-Wharton Conference (2010): 2.

Novy-Marx, R., \& Rauh, J. (2009). The Liabilities and Risks of State-Sponsored Pension Plans. Journal of Economic Perspectives, (2009), 191 - 210.http://dx.doi.org/10.1257/jep.23.4.191

Novy-Marx, R., \& Rauh, J. (2011). Public Pension Promises: How Big Are They and What Are They Worth? Journal of Finance, 66 (4), 2011, 1211-1249.http://dx.doi.org/10.2139/ssrn.1352608 
Pew Center on the States. (2011). The Widening Gap: The Great Recession's Impact on State Pension and Retiree Health Care Costs. April 2011.

Poterba, J., \& Rueben, K. (1999). State fiscal institutions and the U.S. municipal bond market. Book chapter. Fiscal Institutions and Fiscal Performance. University of Chicago Press.http://dx.doi.org/10.3386/w6237

Public Fund Survey. (2013). (http://www.publicfundsurvey.org/publicfundsurvey/scorecard.asp)

Rauh, J. (2010). Are State Public Pensions Sustainable? Why the Federal Government Should Worry about State Pension Liabilities. Working paper, Northwestern University. http://dx.doi.org/10.2139/ssrn.1596679

Rauh, J. (2011). Kellogg Insight Presents. Retrieved March 18, 2012, from "Shortfall for State and Local Pension Systems Today: Over $\quad \$ 4$ Trillion": http://kelloggfinance.wordpress.com/2011/10/06/shortfall-for-state-and-local-pensionsystems-today-over-4-trilli on/

Rauh, J. (2011). Congressional Testimony on Feb. 24, 2011 for the hearing on "The Role of Public Employee Pensions in Contributing to State Insolvency and the Possibility of a State Bankruptcy Chapter" (http://judiciary.house.gov/hearings/pdf/Rauh02142011.pdf).

Rauh, J. (2011). Kellogg Insight Presents. Retrieved March 18, 2012, from "Shortfall for State and Local Pension Systems Today: Over $\quad \$ 4 \quad$ Trillion": http://kelloggfinance.wordpress.com/2011/10/06/shortfall-for-stateand-local-pension-systems-today-over-4-trilli on/.

Standard \& Poors. (2012). U.S. Local Governments: Methodology And Assumptions: http://www.standardandpoors.com/spf/ratings/US_LocalGov_Methodology_Assumptions_3_6_12.pdf

Wang, J., Wu, C., \& Zhang, F. (2008). Liquidity, default, taxes, and yields on municipal bonds. Journal of Banking and Finance 32 (6), 1133-1149. http://dx.doi.org/10.1016/j.jbankfin.2007.09.019

Wilshire Consulting. (2012). Wilshire Report on State Retirement Systems: Funding Levels and Asset Allocation. Santa Montica, CA: Wilshire Associates Incorporated, 2012.

\section{Notes}

Note 1. See the Public Fund Survey (http://www.publicfundsurvey.org/publicfundsurvey/scorecard.asp), jointly sponsored by the National Association of State Retirement Administrators and the National Council on Teacher Retirement.

Note 2. The difference of estimated pension shortfall between different methods is mainly due to the discount rate (or the denominator) used to calculate the present value of projected total future pension benefits. State governments and the Public Fund Survey use actuarial rate (based on pension asset long-term expected investment return), which is generally $8 \%$, as a discount rate, while economists prefer to use Treasury yield, which is much less than the actuarial rate, as a discount rate. High (low) discount rate translates to low (high) pension liabilities. Also see Congressional Testimony by Joshua Rauh, Feb. 24, 2011 for the hearing on "The Role of Public Employee Pensions in Contributing to State Insolvency and the Possibility of a State Bankruptcy Chapter" (http://judiciary.house.gov/hearings/pdf/Rauh02142011.pdf).

Note 3. Source: The National Association of State Retirement Administration's Web site, http://www.nasra.org.

Note 4. As another example, a $\$ 650$ million shortfall of Pittsburg's pension system dried up "funds for the sustained investments that remade Pittsburgh after the 1980s collapse of the steel industry" (Green, 2011, p1). In order to deal with this situation, Mayor Luke Ravenstahl created a 1\% fair-share tax for the privilege of attending colleges in the city of Pittsburgh (Maher, 2009).

Note 5. Note that although there are no formal laws regulating state government financial information disclosure, both Securities Exchange Commissions and Government Accounting Standard Board have specific requirements on filing state government financial report.

Note 6. See "Recent trends in municipal continuing disclosure activities" (DPCDTATA, 2011) (http://www.dpcdata.com). The rate of failure to file current fiscal disclosure further rose to $40 \%$ in 2009.

Note 7. The data are available at http://www.unionstats.com and are constructed by Barry Hirsch and David Macpherson based on the Current Population Survey (CPS), a monthly household survey (Hirsch and Macpherson 2003). 